\title{
A IMPORTÂNCIA ESPACIAL DA PROXIMIDADE GEOGRÁFICA NA PESQUISA CIENTÍFICA E NOS PROCESSOS DE INOVAÇÃO TECNOLÓGICA
}

\author{
THE SPACE IMPORTANCE OF GEOGRAPHICAL PROXIMITY IN SCIENTIFIC \\ RESEARCH AND TECHNOLOGICAL INNOVATION PROCESSES
}

\author{
IMPORTANCE SPATIALE DE LA PROXIMITÉ GÉOGRAPHIQUE \\ DANS LA RECHERCHE SCIENTIFIQUE ET LES \\ PROCESSUS D'INNOVATION TECHNOLOGIQUE
}

Allison Bezerra Oliveira - Universidade Estadual da Região Tocantina

do Maranhão - Imperatriz - Maranhão - Brasil

allisonbzr@gmail.com

\section{Resumo}

0 presente artigo tem como objetivo analisar a importância da proximidade geográfica na pesquisa científica e nos processos de inovação tecnológica mediante os contornos tecnológicos comunicacionais. Desta forma, estabeleceu-se o seguinte questionamento: diante da difusão do uso da comunicação instantânea, a proximidade geográfica na pesquisa científica e inovação ainda é relevante para aqueles que cooperam em pesquisas? Nesse contexto, delimitou-se 0 estado de Pernambuco como recorte espacial, a área de Farmácia como amostragem, e o período de 2000 a 2010 como recorte temporal. Além de reflexão teórica sobre 0 tema, foram mapeadas as conexões entre pesquisadores e a produção de conhecimento deles e, ainda, enviados questionários eletrônicos para os pesquisados. 0s resultados obtidos reforçam a relevância da proximidade geográfica como componente fundamental na construção de relações de cooperação mais duradouras e com maior possibilidade de retorno entre os pesquisadores.

Palavras-chave: Proximidade Geográfica. Pesquisa Científica. Inovação Tecnológica. Pernambuco.

\section{Abstract}

The main goal of this article is to identify the how important is the geographical proximity during the technological innovation process using the communication technologies. To achieve this the following questionnaire were created: in the scenario of instant communication process, is the geographical proximity relevant to the cooperation in the scientific research and innovation? In this context, was used the data from Pernambuco State in the pharmaceutical field between the years of 2000 and 2010 . Beyond the theoretical study, was mapped the connection between the researchers, their knowledge production, and the answer that they provided by electronical questionnaire. The results reinforce the fundamental relevance of geographical proximity during the construction of long term cooperation with greater return between the researchers Keywords: Geographic Proximity. Scientific research. Tecnologic innovation. Pernambuco.

\section{Resumé}

Cette étude a pour but d'analyser l'importance de la proximité géographique au sein du monde de la recherche dans le contexte actuel d'utilisation des nouvelles technologies d'information et de communication. Ainsi, nous avons posé la question suivante: dans un monde où la communication instantanée et à distance se veut toujours plus commode et efficace, comment expliquer l'intérêt de la proximité géographique entre les individus impliqués dans la recherche scientifique? Pour mesurer la légitimité de cette proposition, nous avons envoyé des questionnaires électroniques aux chercheurs du milieu pharmaceutique du Pernambouco actifs de 
2002 à 2010, grâce auxquels nous avons pu cartographier connexions entre eux. Les résultats obtenus ont montré l'importance de la proximité géographique en tant que composante fondamentale pour intensifier les rapports entre chercheurs, donnant ainsi plus de possibilités de retour réflexif sur une science en construction. Mots clés: Proximité géographique. Recherche scientifique. Innovation technologique. Pernambuco.

Introdução

A revolução tecnológica tem possibilitado gradativamente ao longo dos anos um aumento generalizado da mobilidade de pessoas, energia, informação, ideias, bens, usos, produtos e serviços. Esse movimento possibilita a instantaneidade, diminui as noções tempo-espaciais e assegura aos centros de decisão um real poder sobre os outros pontos do espaço.

Tal processo, que corresponde à própria natureza do capitalismo, acelerou-se para hoje alcançar o seu ápice, com a predominância em toda parte de um único sistema técnico, ou, nas palavras de Santos (2006, p. 128), de uma unicidade técnica que é base material da globalização. Com a emergência do meio técnico-científico-informacional, o atual sistema técnico se tornou comum a todas as civilizações, culturas e sistemas políticos, a todos os continentes e lugares, mesmo que sua difusão e inserção ocorram em escalas desiguais (Santos, 2006).

Tal unicidade técnica tem proporcionado o que Santos (2006) chama de unicidade de momentos, e Harvey (2008) chama de compressão tempoespacial. Em ambas as reflexões, é inegável a participação efetiva das tecnologias de informação, processamento e comunicação. A informação ganhou a possibilidade de fluir instantaneamente, sem nenhuma defasagem, comunicando para quase todos os lugares. Embora ocorra de maneira desigual, tal processo desencadeou aumento significativo e gradativo nos processos sociais e relacionais; a expressão máxima dessas transformações é a expansão das redes.

O debate das redes chega a ser onipresente quando falamos nos contornos atuais possibilitados pelos avanços tecnológicos da comunicação e do transporte. A lógica viária fortemente ancorada nos fluxos tem proporcionado gradativa diminuição de algumas rugosidades espaciais e expressivas reflexões sobre a rigidez física da distância. Tais transformações têm sido apropriadas pela pesquisa científica e consequentes processos de inovação tecnológica à medida que as telecomunicações possibilitam o estabelecimento de intercâmbio acadêmico com pessoas distantes geograficamente. 
Contudo, embora sejam inegáveis as contribuições das telecomunicações e da telemediação eletrônica nos processos de cooperação científica, muitas reflexões têm ao longo dos últimos anos apresentado concepções de que tais tecnologias estariam cada vez mais diminuindo a geograficidade dos processos sociais cotidianos, das rugosidades, das proximidades. Em outras palavras, estariam substituindo qualidades formais do espaço geográfico, como a proximidade geográfica.

Nesse contexto, o presente artigo apresenta uma análise teóricoconceitual de tais dinâmicas, além de refletir sobre a relevância ou não da proximidade geográfica nos processos de pesquisa e produção de conhecimento, mesmo em face do advento tecnológico. Dessa forma, pretende-se elucidar uma questão principal: diante da difusão do uso da comunicação instantânea, a proximidade geográfica na pesquisa científica e em processos de inovação ainda é relevante para aqueles que cooperam?

\section{Informação, Conhecimento, Inovação}

A produção, aquisição e difusão do conhecimento são vistas por muitos, cada vez mais, como as características fundamentais da dinâmica competitiva e emancipadora em diversas áreas do conhecimento. Contudo, muito antes desse debate se tornar popular, os estudiosos já haviam manifestado um profundo interesse em distinguir diferentes tipos de conhecimento (Gertler, 2003).

Autores como Polanyi $(1958,1966)$ distinguiram dois tipos de conhecimento: um que poderia ser efetivamente expressado usando formas simbólicas de representação - explícitas ou codificadas - e outro, o conhecimento tácito ${ }^{1}$, expresso em formas de conhecimento que desafiam essa representação formal.

Embora informação e conhecimento estejam correlacionados, não são sinônimos. Também é necessário distinguir dois tipos de conhecimentos: os conhecimentos codificáveis - que, transformados em informações, podem ser reproduzidos, estocados, transferidos, adquiridos, comercializados etc. - $\mathrm{e}$ os conhecimentos tácitos. Para estes a transformação em sinais ou códigos é extremamente difícil já que sua natureza está associada a processos de aprendizado, totalmente dependentes de contextos e formas de interação sociais específicas. (Lastres; Ferraz, 1999, p. 30). 
O conhecimento tácito tem sido reconhecido como componente central da economia da aprendizagem e a chave para a inovação e também como determinante principal da geografia da atividade produtora de conhecimento e inovação, uma vez que o seu papel central no processo de aprendizagem através de interação e consequente acesso à informação e conhecimento, tende a reforçar em muitas circunstâncias o papel do local sobre o global.

Para um número crescente de estudiosos, isso explica a perpetuação e o aprofundamento da concentração geográfica em um mundo cada vez mais fluído, enfraquecendo em alguns momentos, as tecnologias de informação e comunicação cada vez mais baratas e mais difundidas (Polanyi, 1966, 1987; Gertler, 1995, 2003).

Já a informação, pode ser entendida do ponto de vista funcional como um recurso redutor de incertezas. Ela faz parte dos vários símbolos, dados e signos compartilhados diariamente nas socializações e comunicações humanas, sendo também entendida como componente fundamental e anterior na produção de qualquer conhecimento. $\mathrm{O}$ "acesso às informações o primeiro passo para a comunicação que propiciará o compartilhamento e intercâmbio de resultados de pesquisas e que confere às comunidades a oportunidade efetiva de participação na sociedade da informação" (Fujino et al., 2009, p. 215)

Para Lastres e Albagli (1999), o novo papel da informação e do conhecimento no mundo contemporâneo vem provocando modificações significativas nas relações, na forma e no conteúdo do trabalho, o qual assume um caráter cada vez mais "informacional”. Logo, as capacidades de transporte, transferência, produção e aquisição de informação e conhecimento se tornam aspectos centrais na produção de recursos materiais e imateriais necessários à manutenção de uma série de necessidades sociais. Em uma sequência simples e sucinta, podemos dizer que a informação contribuiria para a construção de conhecimento, que, por sua vez, é peça fundamental para a produção da inovação. 
Figura 1 - Informação, conhecimento e inovação

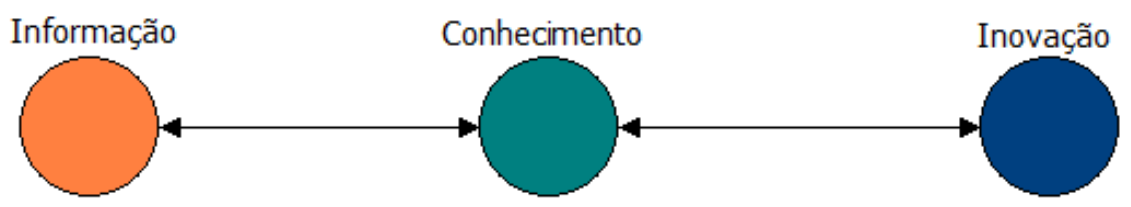

Fonte: 0 autor (2018).

A inovação é entendida como resultado de uma ação coletiva e sistêmica na qual interagem diversos panoramas (científico, educacional, tecnológico, técnico, econômico, social e institucional - formalizado ou não) que permitem a empresas, organizações e pessoas aprenderem, usarem e acumularem capacidades e competências, além de desenvolverem novos produtos, bens e processos. Evidenciando, assim, de acordo com Fernandes (2004), não só a produção de novas tecnologias e a descoberta de novos materiais e/ou produtos, mas igualmente a adoção, tanto pela empresa quanto pelo Estado e sociedade civil, de um novo processo e de novas práticas organizacionais. Para tal, a inovação depende fortemente da capacidade de trocar informação, produzir e absorver conhecimento, sobretudo conhecimento científico.

Fernandes e Lima (2006) afirmam que a inovação é um processo: (1) coletivo; (2) interativo; (3) cumulativo; (4) não linear; e (5) sistêmico. É coletivo, visto que, na atualidade, são muitos os agentes envolvidos e não um inventor isolado; interativo porque a troca de conhecimento entre diferentes agentes envolvidos no desenvolvimento da inovação é fundamentalmente indispensável para que ele ocorra, em vista da impossibilidade de um único agente deter todo o conhecimento e todas as informações necessárias ao processo; cumulativo, pois o conhecimento acumulado propicia a base para o desenvolvimento de novo conhecimento ao longo do tempo; não linear, porque se processa em várias etapas, e não necessariamente em sequência do laboratório à fábrica; e sistêmico porque o processo de inovação resulta da ação de vários agentes e das relações entre eles. 
Cassiolato e Lastres (2005) argumentam que os principais pressupostos da inovação seriam, portanto: (A) o conhecimento é a base do processo inovador, e sua criação, uso e difusão alimentam a mudança econômica, constituindo-se em importante fonte de competitividade sustentável, associando-se às transformações de longo prazo na economia e na sociedade; (B) o aprendizado é o mecanismo-chave no processo de acumulação de conhecimentos; (C) a empresa é considerada o ponto mais importante nesse processo. Porém, o processo de inovação é geralmente interativo, contando com a contribuição de vários atores, detentores de diferentes tipos de informações e conhecimentos, dentro e fora da empresa; (D) os processos de aprendizado, capacitação e inovação são influenciados e influenciam os ambientes socioeconômicos e políticos onde se realizam.

Desta forma, haveria uma ampla gama de informações e conhecimentos essenciais que favorecem a geração e incorporação de inovações. Tais processos e seus elementos, são constituídos a partir de uma gama contínua de tentativas, erros, feedbacks, assimilação, uso e difusão, que necessitam de cooperação constante e em muitos casos, de proximidade geográfica.

\section{A importância espacial da proximidade geográfica}

Cabe empreender reflexão teórico-conceitual acerca da discussão sobre a importância da proximidade na ciência geográfica. As ideias aqui apresentadas corroboram com os debates desenvolvidos por autores como Polanyi (1958, 1966, 1987), Gertler (1995, 2003), Allen (2000), Storper e Venables (2001), Torre e Rallet (2005), Lagendijk e Lorentzen (2007).

O primeiro ponto fundamental é o que consideramos como proximidade geográfica. A "proximidade" aqui mencionada é usada tanto no sentido literal, no que se refere à distância geográfica, como no sentido espacial de compartilhamento de língua comum, modos de comunicação, estrutura de trabalho, costumes, convenções, regionalismos, rotinas, simbolismos e normas sociais compartilhadas.

Ou seja, a proximidade como continuidade espacial, dentro de um contexto histórico e geográfico constante de (re)produção das relações sociais cotidianas. Do ponto de vista das construções e produções acessíveis e cotidianas, Lagendijk e Lorentzen (2007, p. 460) destacam que fundamentalmente a proximidade geográfica não deve ser equacionada apenas como proximidade física, pura e simplesmente. 
Pelo contrário, é um conceito dinâmico, intimamente associado com a noção de estruturação espacial. Por um lado, a proximidade geográfica é um produto de uma construção histórica, de um acumulado de infraestruturas de transporte e de locais de encontro, tanto de uma forma e sentido mais material e virtual, bem como, gostaríamos de acrescentar, a formação de espaços territorialmente delimitados, dimensões sociais, institucionais, políticas e econômicas. (Lagendijk; Lorentzen, 2007, p. 460).

Para Lagendijk e Lorentzen (2007), a proximidade geográfica promove e potencializa também efeitos casuais não esperados, além de sustentar a conectividade e posicionalidade dos agentes. Da mesma forma, Gertler (1995) destaca a importância espacial como componente na facilitação do processo de compartilhamento de códigos de comunicação e cooperação, bem como na construção de um legado comum de práticas e instituições sociais. Ainda segundo o autor, a proximidade parece facilitar a formação e manutenção em alta qualidade de relacionamentos que necessitem de intensiva interação e proporciona maior e mais livre fluxo de informações entre os atores.

Para ele, no caso em que grandes distâncias venham a intervir, problemas podem daí decorrer, mesmo com a funcionalidade eletrônica. A "falta de proximidade" tenderia a tornar o processo de cooperação oneroso ${ }^{2}$; e mesmo (in)voluntariamente os membros ainda tendem a procurar o estabelecimento de conexões com outros que estão próximos.

As ideias de Gertler (1995) são reforçadas por Lagendijk e Lorentzen (2007, p. 462) ao destacarem que "os lugares continuam sujeitos a formas mais simbólicas de posicionalidade”. Essa compreensão nos levaria ao nosso segundo ponto fundamental de entendimento aqui apresentado, que trata da importância e impacto das multiplicidades espaciais existentes e fortemente construídas cotidianamente, que variavelmente podem se sobrepor às relações telemediadas.

Na mesma linha de raciocínio, Allen (2000) evidencia que as rotinas organizacionais em curso e as práticas sociais de trabalhos coletivos envolvidos em empreendimentos comuns são mais facilmente fortalecidas pelas interações cotidianas. Para tal, as práticas espaciais produzem perspectivas que em alguns casos - ou momentos - são permitidas à distância, embora mantenham significativa importância presencial.

Portanto, estar presencialmente em dados momentos com os membros de uma rede não só facilita a interação, a coordenação e a 
comunicação entre eles, como potencializa os níveis de proximidade social e, consequentemente, uma maior troca e compreensão de códigos e signos, além da construção de laços de confiança. A necessidade de contato face a face é tanto mais importante quanto maior for o caráter tácito do conhecimento.

[...] com relação à troca eletronicamente mediada, a estrutura do contato face a face oferece possibilidade incomum de interrupção, reparo, feedback e aprendizagem. Contrariamente a interações grandemente sequenciais, a interação face a face possibilita a duas pessoas emitir e receber mensagens simultaneamente. $\mathrm{O}$ ciclo de interrupção, feedback e reparo, possível na interação face a face é tão rápido que é praticamente instantâneo. (Nohria; Eccles, 1992, p. 292).

Polanyi (1987, p. 4) afirma que "sabemos mais do que somos capazes de comunicar”. A comunicação face a face manifesta sua riqueza e poder não apenas por nos permitir estar em contato presencialmente e detectar mensagens intencionais, mas também mensagens não intencionais que podem ser decodificadas apenas por esse contato visual.

O contato FaF, elimina o anonimato, faz aumentar a probabilidade de boas escolhas, graduais e interativas, sobre as habilidades dos outros, quando não se pode saber, de início, a respeito de sua capacidade de operosidade; também pode fazer com que os indivíduos se tornem mais capazes de sinalizar para os outros suas habilidades e níveis de operosidade. É uma maneira de tornar transparente e de baixo custo este tipo de informação, embora também permita, nos primeiros contatos $\mathrm{FaF}$, que certas pessoas se juntem e permaneçam no grupo. (Storper; Venables, 2001, p. 41).

Da mesma forma, Polanyi (1987) argumenta que o conhecimento científico é produzido por indivíduos que impregnam sua busca por novos conhecimentos com conteúdos profundamente pessoais, que são resultado de uma série de interações e particularidades. Em outras palavras, o conhecimento científico não seria apenas um conjunto claramente articulado de axiomas, regras, algoritmos e declarações. Esses, por sua vez, em alguns casos, não podem ser compreendidos através de telefonemas, chats, webconferências, e-mails, servidores e demais tecnologias.

Ou seja, mesmo com os meios eletrônicos, o conhecimento não flui tão facilmente. Isso porque a sua transmissão é mais comum por meio 
da interação face a face entre os parceiros que já compartilham questões espaciais "básicas”: mesma língua, “códigos” comuns de comunicação, convenções e normas compartilhadas, conhecimento pessoal de cada um baseado em uma história passada de colaboração de sucesso ou interação informal. Essas semelhanças servem ao propósito vital de construção de confiança entre os parceiros, o que facilita o fluxo local de conhecimento tácito entre eles (Gertler, 1995; Morgan, 2004).

Torre e Rallet (2005, p. 53) também discutem que mesmo com a atual explosão comunicacional, ou "a onipresença promovida pela comunicação e mobilidade - física e virtual”, certos recursos e relações permanecem espacialmente enraizados. Afirmam ainda que a proporção de enraizamento da presencialidade de contatos face a face varia de acordo com a atividade, "mas que em todas, ainda encontraríamos faísca da necessidade da produção espacial presencial cotidiana".

Portanto, a proximidade virtual proporcionada pelos fluxos tecnológicos não pode substituir a proximidade geográfica nas transações de alta complexidade e ambiguidade, por exemplo, não só no âmbito do próprio funcionamento da pesquisa científica, mas na própria construção e consolidação de relações que necessitem de circunstâncias presenciais.

A presença mútua - estar suficientemente próximo, a ponto de tocar-se um no outro - permite o "contato" visual e a "proximidade emocional”, a base para a construção das relações humanas. A comunicação FaF, mais que apenas uma troca, é um desempenho pelo qual o discurso e outros tipos de ações e contextos se juntam para exercitar a comunicação, de uma maneira muito complexa, em muitos e diferentes níveis ao mesmo tempo. (Storper; Venables, 2001, p. 32).

Para eles, a possibilidade dos pesquisadores "estarem aqui" e se comunicarem face a face em dados momentos não só é necessário, como reduz uma série de incertezas, e dinamiza o trabalho cooperativo. Em outras palavras, ter os demais membros "próximos" na hora de se formar uma equipe ou durante a realização do projeto facilita a realização do trabalho em si, além da inserção de novos membros no grupo. 


\section{Questões metodológicas}

Na busca de literatura sobre o tema que destaca a relevância espacial da proximidade, utilizou-se, além da Geografia, mecanismos da pesquisa em Ciência da Informação e análise de redes sociais. Focou-se inicialmente em informações contidas em publicações científicas e técnicas, envolvendo essencialmente artigos, livros e patentes (registradas ou depositadas), uma vez que são fontes materiais confiáveis de produção de conhecimento. Desta forma, são a base e, em muitos casos, o resultado de processos de inovação tecnológica. Para tal, utilizou-se a grande área da Saúde e, em específico, a subárea de Farmácia, como recorte de amostragem para a pesquisa. A escolha da área de Farmácia deu-se por dois motivos principais: a) pela importância dessa área na superação de desigualdades sociais no âmbito da saúde; e b) por tal área ser considerada na literatura evolucionária sobre conhecimento e inovação tecnológica (Pavitt, 1984) como essencialmente dependente da troca de conhecimentos com outras áreas na pesquisa científica básica, com Biologia, Química, Física, Biomedicina, Medicina, Engenharia, Biotecnologia, dentre outras, o que a torna extremamente dinâmica em processos de inovação.

Posteriormente, utilizou-se enquanto recorte temporal o período de 2000 a 2010 para fins de mapeamento e os censos do CNPq de 2000, 2005 e 2010 para identificação do quantitativo e evolução de grupos de pesquisa e pesquisadores da grande área de Farmácia no estado de Pernambuco ${ }^{3}$. Foram verificados os artigos que incluíam tais pesquisadores como (co) autores, que fossem oriundos de pesquisas cooperativas e publicados e evidenciados na Plataforma Lattes. Para os trabalhos publicados verificou-se a ocorrência de processos de inovação que resultaram em depósitos de patentes no Instituto Nacional de Patentes (INPI). Para maior amplitude de informações sobre a pesquisa científica, utilizou-se três bases de dados indexadas: ISI Web of Knowledge, SciVerse Scopus e Scientific Electronic Library Online (SciELO). O intuito foi também mapear autores que não aparecessem em grupos de pesquisa, mas que desenvolvessem pesquisas em conjunto. Eliminando-se as repetições, chegou-se ao número de 14 líderes de grupos de pesquisa e 72 pesquisadores envolvidos no ano de 2000, 16 líderes de grupos de pesquisa e 118 pesquisadores envolvidos no ano de 2005, e 24 líderes de grupos e 382 pesquisadores envolvidos no ano de 2010 na base de dados. 
Essas informações foram transformadas em três matrizes no Excel referentes a cada ano pesquisado, que é reflexo numérico das relações expostas nos artigos científicos, e essas, por sua vez, serviram como modelo para que as redes de pesquisa científica fossem ao final mapeadas. Após a construção da matriz numérica, os dados foram migrados para os softwares Ucinet ${ }^{4}$ e Netdraw que criam o desenho final. Tal processo foi desenvolvido a partir da metodologia trabalhada por Newman (2001), aperfeiçoado pelos pesquisadores da Ciência da Informação e pôde ser melhor detalhado na Figura 2 a seguir.

Figura 2 - Processo de mapeamento de rede científica com base em artigos publicados

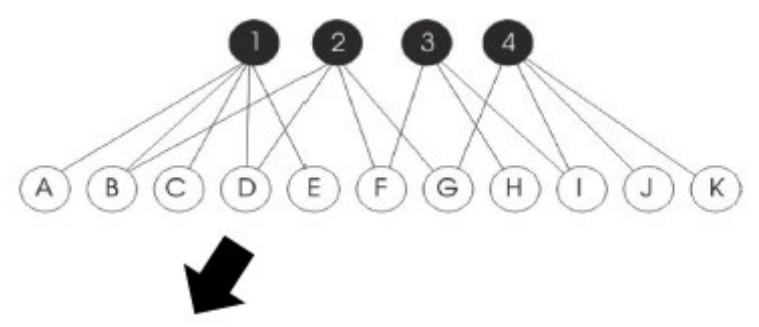

\begin{abstract}
A B C D E F G H I J K
\end{abstract}
A 111111111000000000
B $0 \begin{array}{llllllllllllllllllll} & 1 & 1 & 1 & 1 & 1 & 1 & 0 & 0 & 0 & 0\end{array}$
C 1100111110000000
D 111100111111100000
E 111111010100000000
F $\begin{array}{lllllllllllll}0 & 1 & 0 & 1 & 0 & 0 & 1 & 1 & 1 & 0 & 0\end{array}$
G $0 \begin{array}{lllllllllll} & 1 & 0 & 1 & 0 & 1 & 0 & 0 & 1 & 1 & 1\end{array}$

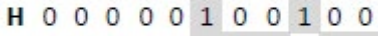
I 00000001111011
J $0 \begin{array}{lllllllllllllllll}1 & 0 & 0 & 0 & 0 & 0 & 1 & 0 & 1 & 0 & 1\end{array}$
K $00 \begin{array}{lllllllllllllllllll} & 0 & 0 & 0 & 0 & 0 & 1 & 0 & 1 & 1 & 0\end{array}$

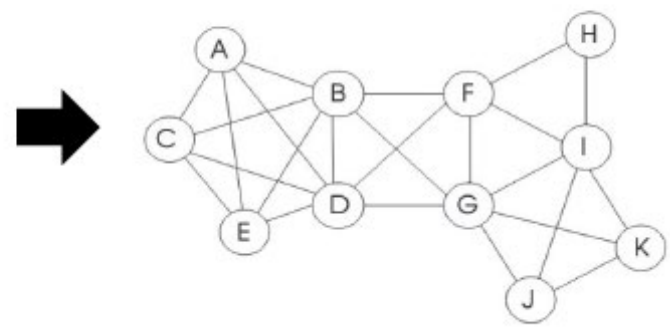

Fonte: Oliveira (2014), a partir de Newman (2001) e Balancieri et al. (2005).

Nela, os números de 1 a 4 são os artigos publicados, e os pesquisadores são as letras compreendidas entre A e K. A partir das conexões entre os autores, por meio de suas relações na pesquisa científica, gera-se uma matriz relacional, onde 1 significa que existe conexão entre dois atores, e 0 que não existe conexão. A matriz é o reflexo numérico das 
relações expostas nos artigos científicos e serve como modelo para que a rede seja ao final mapeada. Na rede mapeada, os traços são conexões e os pontos são pesquisadores, que foram definidos de acordo com sua localização ${ }^{5}$ geográfica, discriminada no artigo científico na data da publicação.

Tais informações, apresentadas nos mapeamentos, foram consolidadas com questionários eletrônicos enviados aos principais pesquisadores que aparecem na pesquisa (líderes de grupos de pesquisa e pesquisadores que aparecem em pelo menos dois anos mapeados) e obteve-se $67 \%$ de retorno dos questionários.

Proximidade geográfica e pesquisa científica em inovação tecnológica

É plausível que no processo de mapeamento das relações de pesquisa científica e processos de inovação tecnológica, que tem Pernambuco como ponto de partida, encontre-se uma predominância de membros desse estado. Contudo, é de se considerar que sejam encontrados pesquisadores de diversos espaços geográficos, uma vez que, como já abordado neste trabalho, são inegáveis as contribuições das telecomunicações no processo de compartilhamento e troca de ideias, além do próprio trabalho à distância.

É esperado também que ao longo dos anos, nos três períodos estudados, exista aumento progressivo no quantitativo de pesquisadores trabalhando em conjunto não só mediante as perspectivas de trabalho cooperativo à distância, mas também pelo fato de que o Brasil viveu - em especial no período compreendido entre 2003 e 2015 - aumento gradativo dos investimentos em CT\&I. Na Figura 3, expressa a seguir, podemos observar os primeiros resultados compreendidos nos anos de 2000 (à esquerda) e 2005 (à direita). 
Figura 3 - Mapa das conexões de pesquisa científica na área de Farmácia, em Pernambuco, nos anos de 2000 e 2005
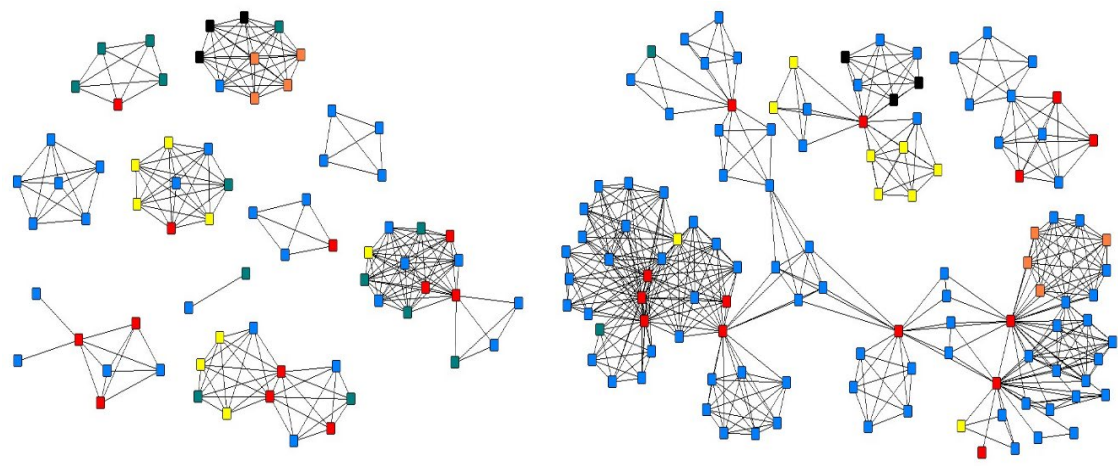

Fonte: 0 autor (2018).

No ano de 2000 foram mapeados 72 pesquisadores; desses, 79\% (57) localizavam-se em Pernambuco, mais precisamente na Região Metropolitana de Recife, 11\% (8) eram estrangeiros, 4\% (3) encontravam-se na região Centro-Oeste e 6\% (4 pesquisadores) no estado da Paraíba. No ano de 2005, houve expressivo aumento de agentes envolvidos em processos de pesquisa, o que representou 118 pesquisadores mapeados, estando 2\% (3 pesquisadores) deles localizados no Centro-Oeste; 3\% (4 pesquisadores) em outros estados do Nordeste, especificamente na Paraíba; e 8\% (9 pesquisadores) fora do Brasil. Os demais 102 pesquisadores (87\%) estavam concentrados, sobretudo, em Pernambuco, na região metropolitana de Recife, assim como em 2000. Quanto à maioria desses agentes, há, notadamente, intensa concentração geográfica de pesquisadores na Região Metropolitana de Recife (evidenciada pelos pontos vermelhos e azuis), onde há também maior aglomeração de instituições de pesquisa.

No processo de pesquisa, uma série de competências e formações diversas são necessárias. Cooperação e compartilhamento de conhecimentos complementares, além de equipamentos, compactuam para formar uma cadeia de pesquisa científica que necessita de firme cooperação e engajamento para que um novo conhecimento ou inovação tecnológica seja produzido. Nesse processo, a proximidade geográfica 
apresenta a possibilidade de maior segurança no comprometimento e participação efetiva nas atividades, além de barateamento da produção, à medida que permite a consolidação de práticas comuns a todos aqueles envolvidos. Tal perspectiva pode ser visualizada a partir do mapeamento dessas relações para o ano de 2010, expresso na Figura 4.

Figura 4 - Mapa das conexões de pesquisa científica na área de Farmácia, em Pernambuco, em 2010

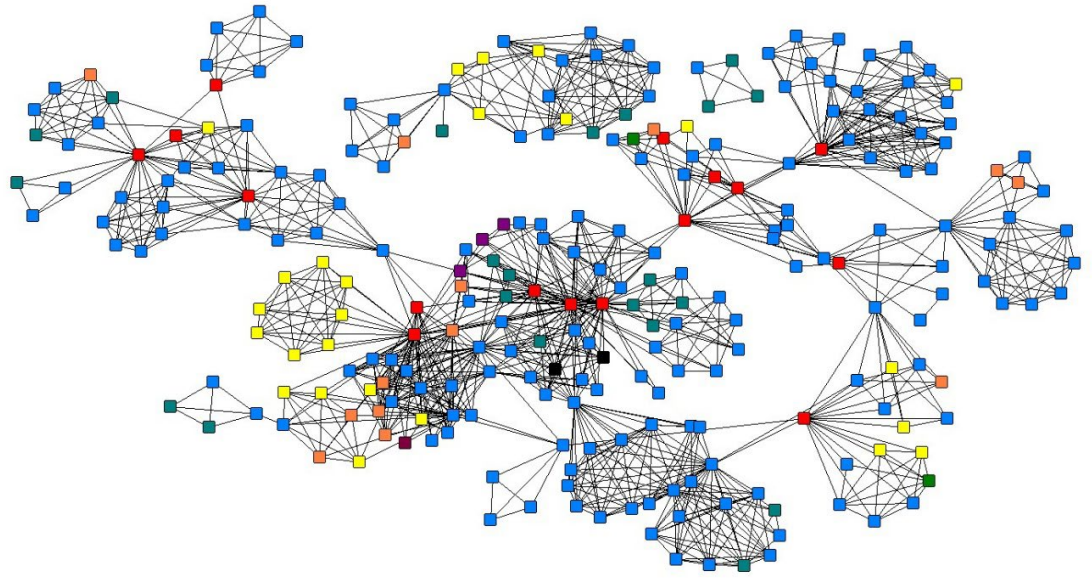

Fonte: 0 autor (2018).

Nela, reproduzindo comportamentos apresentados nos anos de 2000 e 2005, encontra-se intensa concentração geográfica no estado de Pernambuco (pontos vermelhos e azuis); ao todo, dos 382 pesquisadores mapeados, 84\% (318) encontram-se em Pernambuco e, em especial, com total concentração na Região Metropolitana da capital Recife (RMR).

As relações estabelecidas são quase exclusivamente em formato circular, em torno dos líderes dos grupos de pesquisa (pontos vermelhos), o que indica que o sistema de objetos da produção científica na área de Farmácia é significativamente fixo no estado e funciona em torno dos grupos de pesquisa (que detêm, além do suporte técnico para a pesquisa, também o espaço corriqueiro de contato presencial). Os grupos de pesquisa se tornam suportes cotidianos na construção das relações de 
pesquisa, mesmo que em diversos momentos sejam utilizados mecanismos eletrônicos para a comunicação e a delegação de tarefas.

A concentração geográfica de pesquisadores, mais do que ser fruto de uma necessidade de proximidade geográfica no processo de pesquisa pernambucana na área de Farmácia, nos mostra que essa proximidade é fundamental para que as relações de cooperação na pesquisa possam consolidar ações e objetos cotidianos, fixos e móveis, que estejam a serviço dessa dinâmica e sejam acessíveis. Em outras palavras, os líderes de grupos de pesquisa que detêm o capital de financiamento, além dos laboratórios, são membros fundamentais para a permanência e a circulação de pessoas nesse emaranhado de conexões.

Quando olhamos a participação quantitativa de pesquisadores de outros estados, temos 19 (5\%) em outros estados do Nordeste, 3 (1\%) na região Centro-Oeste, 12 (3\%) no Sudeste, 8 (2\%) no Sul do Brasil, e 22 (6\%) estão em outros países. Todos os pesquisadores encontrados nas regiões Nordeste e Centro-Oeste executaram atividades no passado no estado de Pernambuco e construíram vínculos profissionais duradouros; pelo menos metade destes já realizaram atividades de pesquisa conjuntamente no período de 2000 a 2010 . O mesmo ocorre com pesquisadores estrangeiros que já apresentam histórico de participação anterior.

As concentrações geográficas apresentadas nos dados apontam para uma proximidade que ultrapassa a mera distância em quilômetros e diz respeito à concentração em um mesmo estado e à grande centralidade apresentada pelos líderes dos grupos de pesquisa e suas instalações. Apontam também para o fato de que ainda são mais valorizadas interações e cooperações não planejadas - frequentes e eficazes - mais amplas, tais como compartilhamento de idioma comum, regionalismos, estruturas e regimes de trabalhos, convenções e outros simbolismos mais facilmente apreendidos por quem "está aqui".

Quando verificados os processos que originaram patentes, frutos de inovações tecnológicas no período de 2000 a 2010, foram encontradas 12 patentes registradas no INPI e mais 21 pedidos depositados entre 2011 e 2013, resultados de pesquisas realizadas entre 2005 e 2010. Nesses, excluindo as repetições, haviam 32 pesquisadores envolvidos, dos quais apenas dois não residiam em Pernambuco. A concentração geográfica é ainda maior quando os resultados geram patentes e novos conhecimentos a serem usados de imediato pela população. 
Em ambos os casos, ou seja, na produção de conhecimento científico e no desenvolvimento de inovação tecnológica, a proximidade geográfica torna-se mais relevante na medida em que acelera o processo de feedbacks, resultados em conjunto e, sobretudo, confiabilidade de informações como registros de patentes. Isso porque o processo de criação de habilitações e as influências da taxa de aperfeiçoamento e inovação são intensivamente locais e, como tal, carregam especificidades do contexto espacial em que são produzidos.

A fim de compreendermos com mais propriedade os dados, para nós, assim como para Gertler (1995), a forma mais direta de tentarmos determinar a importância da proximidade geográfica, do pesquisador "estar aqui" na pesquisa científica - além da própria construção de uma literatura pertinente sobre a temática e os resultados obtidos através do mapeamento - é perguntarmos diretamente para os agentes em questão.

Nesse caso, buscou-se verificar qual a importância atribuída pelos pesquisadores ao fato de ter outros membros "próximos" na hora de se estabelecer e desenvolver uma pesquisa científica cooperativa e processos que podem gerar uma patente. Os principais questionários estão compilados no Quadro 1 abaixo:

Quadro 1 - Questões sobre a importância da proximidade geográfica

\begin{tabular}{|l|l|l|l|}
\multicolumn{2}{|c}{ Questionamento } & Não \\
\multicolumn{2}{|l}{ Irrelevante } \\
\hline Sobre a importância de conhecer pessoalmente o pesquisador & $65 \%$ & $30 \%$ & $5 \%$ \\
\hline Sobre a importância de ter trabalhado anteriormente com o outro & $85 \%$ & $15 \%$ & \\
\hline Sobre a importância de relações orientador x orientando & $95 \%$ & & $5 \%$ \\
\hline Sobre a importância de encontros presenciais na pesquisa em rede & $70 \%$ & $30 \%$ & \\
\hline $\begin{array}{l}\text { Se considera que a comunicação eletrônica é substituta eficaz da comunicação } \\
\text { presencial }\end{array}$ & $30 \%$ & $70 \%$ & \\
\hline Sobre a importância de pesquisar com quem está próximo geograficamente & $95 \%$ & & $5 \%$ \\
\hline $\begin{array}{l}\text { Sobre a importância da proximidade geográfica para processos de inovação } \\
\text { tecnológica }\end{array}$ & $95 \%$ & & $5 \%$ \\
\hline $\begin{array}{l}\text { Sobre a importância da pesquisa científica básica como suporte na geração de } \\
\text { inovações }\end{array}$ & $100 \%$ & & 5 \\
\hline
\end{tabular}

Fonte: 0 autor (2014). 
Quando perguntados sobre a importância de conhecer pessoalmente o pesquisador, a importância de ter trabalhado previamente com o outro na hora de estabelecer uma pesquisa em rede e a importância da relação orientador x aluno, mais de $60 \%$ dos pesquisados responderam "sim" a todas essas perguntas, afirmando se tratarem de fatores importantes. Outro ponto pertinente trata das relações de confiança entre orientador x orientando, que possibilitam a construção de relações de confiança contínuas a partir da formação acadêmica; nesse caso, 95\% dos pesquisadores informaram valorizar tais elementos, e apenas $5 \%$ consideraram irrelevante.

Nesses casos a proximidade geográfica é mais que meramente física, é uma proximidade estabelecida pelas relações de confiança que são mais facilmente constituídas cotidianamente. Para os pesquisadores, essas rugosidades permitidas apenas pelo exercício contínuo e presencial de cooperação são fundamentais para o ingresso e a permanência de membros nas diversas redes estabelecidas. Além de serem fundamentais para o próprio exercício satisfatório da pesquisa científica.

Quando perguntados sobre a importância de encontros presenciais na pesquisa em rede, $70 \%$ dos entrevistados afirmaram valorizar esse tipo de encontro. Nessa mesma percepção espacial do trabalho em rede, 70\% dos entrevistados não consideraram a comunicação eletrônica como substituta eficaz da comunicação presencial. Há, dessa forma, uma inequívoca e clara valorização do contato face a face, que somente é possibilitado pelo "estar aqui". Na mesma perspectiva, houve um significativo percentual de $95 \%$ de respostas positivas quanto à importância de se pesquisar com quem está próximo geograficamente.

Quanto à importância da pesquisa científica básica como suporte na geração de futuras inovações, há consenso reforçando a literatura sobre o papel desta etapa na produção de conhecimento mais consistente. Já em relação à importância da proximidade geográfica nos processos de inovação tecnológica, 5\% consideraram irrelevante (mesmo nessas respostas os pesquisadores foram enfáticos ao dizer que em termos de confiabilidade e processos com maior nível de comprometimento e responsabilidade, a proximidade seria fundamental); a grande maioria (95\%) ratificou o quão é importante a proximidade na construção de resultados profícuos. 


\section{Considerações finais}

O "estar aqui”, que promove a comunicação face a face, também possibilita a redução de incertezas e dinamiza o processo cooperativo. Essa especificidade da proximidade geográfica se relaciona com certas modalidades do exercício de confiança cotidiana, que têm a ver não só com a reputação do outro (o que poderia se realizar, até certo ponto, à distância), mas com práticas mais complexas e mais efetivas a longo prazo. Essa prática constitutiva da confiança se apoia nas relações que só podem ser realizadas pessoalmente.

A pesquisa científica básica e os processos de inovação tecnológica, desta forma, não podem ser compreendidos apenas como um elemento dual: ancorados no suporte tecnológico e no fomento financeiro. As relações sociais conduzidas nas rugosidades cotidianas ainda mantêm importância expressiva dentro do convívio, confiança e formação diária. A concentração geográfica de pesquisadores, repetida em diversos momentos, reforçou isso.

Por isso, é importante a compreensão de que cada lugar tem suas peculiaridades de acordo com seu capital humano, social, histórico e econômico. Logo, todos os lugares, regiões e territórios terão desenvolvimentos diferentes, apresentando características distintas com respeito às competências para inovar, introduzindo-se fatores como cooperação, conhecimento e a própria relevância que é dada à questão da proximidade geográfica.

Dessa forma, a proximidade geográfica abrange as mesmas contradições que produzem o espaço e que também geram as condições de reprodução das relações sociais que, por sua vez, se fortalecem à medida que os atores envolvidos estejam presencialmente "à disposição" para trocas simbólicas, políticas, científicas e econômicas cotidianas.

A importância simbólica da proximidade é reiterada nos questionários na medida em que se pode perceber que relações de confiança, frutos de vínculos de orientação e formação acadêmica, eram extremamente valorizadas e mesmo pesquisadores residentes em outros países, de onde colaboravam à distância, já haviam exercido anteriormente atividades cooperativas presenciais, sendo assim, a experiência importante elemento na permanência de membros nos grupos. Em processos tão sérios de pesquisa, não há estranhos no grupo. 
As discussões acerca das comunicações eletrônicas devem ser relativizadas com mais cautela e olhadas sob múltipla variáveis e espaços geográficos. Isso implica considerarmos que a imersão tecnológica e as práticas cooperativas são frutos de contextos geográficos distintos, de temporalidades científicas, técnicas e, sobretudo, políticas e devem ser olhadas não de uma forma generalista, mas sim extremamente específica.

Por fim, é importante ratificar que existem nuanças da comunicação pessoal que requerem proximidade entre as pessoas, como regionalismos, contextos históricos e linguagens corporais promovidas apenas pelo contato face a face. É imprescindível, portanto, não confundir o alcance espacial com a profundidade das relações sociais, assim como os contextos geográficos distintos, uma vez que o entendimento de linguagens regionais e a difusão de conhecimento em rede - telecomunicações - podem não ser facilmente compreendidos.

\section{Notas}

1 De acordo com Gertler (2003), talvez a maneira mais fácil de definir o conhecimento tácito é especificando o que ele não é. O desempenho de habilidades tais como a natação, o desembarque de um avião, identificar o rosto de uma pessoa, andar de bicicleta ou fazer pão, por exemplo, são da ordem do tácito. Em cada caso, o bom desempenho de uma habilidade depende da observância de um conjunto de regras que não são claramente conhecidas.

20 autor indica alguns problemas tais como: fuso horário, postos de fronteiras, distâncias físicas de deslocamento, diferenças entre infraestruturas técnicas, problemas de comunicação relacionados às diferentes linguagens, idiomas, regionalismos, diferenças contextuais das práticas no local de trabalho, as culturas de formação etc.

3 Diante do volume de dados gerados por uma pesquisa a nível nacional, utilizou-se apenas uma Unidade Federativa brasileira como amostragem, o que, por sua vez, já gerou quantitativo expressivo de informações.

40 Ucinet é um pacote de software para a análise de dados de redes sociais. Foi desenvolvido por Lin Freeman, Everett Martin e Steve Borgatti e inclui a ferramenta de visualização da rede NetDraw.

5 Tanto o Ucinet quando o NetDraw não possuem ferramentas para a criação de legendas para as imagens, o que se torna uma verdadeira limitação. Desta forma, optouse por caracterizar os atores com cores de acordo com a procedência geográfica assim traduzidos: Cor azul, residente em Pernambuco; Vermelha, Líder de grupo de pesquisa residente em Pernambuco; Laranja, outros estados do Nordeste; Verde, região sudeste; Preto, centro oeste; roxo, sul e amarelo, pesquisadores estrangeiros. 


\section{Referências}

ALLEN, J. Power/economic knowledge: symbolic and spatial formations. In: BRYSON, J. et al. (Ed.). Knowledge, Space, Economy. London: Routledge, 2000. p. 15-33.

BALANCIERI, R. et al. Análise de redes de colaboração científica sob as novas tecnologias de informação e comunicação: um estudo na Plataforma Lattes. Ciência da Informação, Brasília, v. 34, n. 1, p. 64-77, jan./abr. 2005. Disponível em: < http://revista.ibict.br/ciinf/article/view/1103/1223 $>$. Acesso em: 24 fev. 2019.

CASTELLS, M. A sociedade em rede. 6. ed. São Paulo: Paz e Terra, 1999. 698 p. (A era da informação: economia, sociedade e cultura, v. 1).

CASSIOLATO, J. E.; LASTRES, H. M. M. (Org.). Conhecimento, sistemas de inovação e desenvolvimento. Rio de Janeiro: Contraponto, 2005.

Inovação e sistemas de inovação: relevância para a área de saúde. $\bar{R} \bar{E} I I \bar{S}-$ R. Eletr. de Com. Inf. Inov. Saúde, Rio de Janeiro, v. 1, n. 1, p. 153-162, jan./jun. 2007. Disponível em: <https://www.reciis.icict.fiocruz.br/index.php/ reciis/article/view/890/1535 >. Acesso em: 06/01/2018.

FERNANDES, R. J. G. Dinâmicas industriais, inovação e território: abordagem geográfica a partir do Centro Litoral de Portugal. Coimbra: Fundação Caloustre Gulbenkian, 2004.

FERNANDES, A. C. de A.; LIMA, J. P. Cluster de serviços: contribuições conceituais a partir de evidências do polo médico do Recife. Revista Nova Economia, Belo Horizonte, v. 16, p.11-47, 2006. Disponível em: <http://www.scielo.br/pdf/neco/ v16n1/v16n1a01.pdf $>$. Acesso em: 17/05/2018.

FUJINO, A. et. al. Políticas públicas de incentivo à formação de redes sociais e colaborativas em ciência e tecnologia. In: POBLACIÓN, D. A.; MUGANAINI, R.; RAMOS, L. M. S. V. C. (Org.). Redes sociais e colaborativas em informação científica. São Paulo: Angellara, 2009. 653 p.

GERTLER, M. S. Tacit knowledge and the economic geography of context, or The undefinable tacitness of being (there). Journal of Economic Geography, v. 3, n. 1, p. 7599, 2003. Disponível em: < https://doi.org/10.1093/jeg/3.1.75 > . Acesso em: 25/01/2018.

. "Being there": proximity, organization, and culture in the development and adoption of advanced manufacturing technologies. Economic Geography, v. 71, n. 1, p. 1-26. 1995. Disponível em: < https://www.jstor.org/stable/144433>. Acesso em: 25/01/2018.

HARVEY, D. Condição Pós-Moderna. 17. ed. São Paulo: Edições Loyola, 2008. $349 \mathrm{p}$.

LAGENDIJK, A.; LORENTZEN, A. Proximity, Knowledge and Innovation in Peripheral Regions: On the Intersection between Geographical and Organizational Proximity. European Planning Studies, v. 15, n. 4, Apr. 2007. Disponível em: < https://doi.org/10.1080/09654310601133260> Acesso em: 20/10/2017. 
LASTRES, H. M. M.; ALBAGLI, S. Chaves para o terceiro milênio na era do conhecimento. In: . (Org.). Informação e globalização na era do conhecimento. Rio de Janeiro: Editora Campus, 1999. p. 7-26.

LASTRES, H. M. M.; FERRAZ, J. C. Economia da informação, do conhecimento e do aprendizado. In: LASTRES, H. M. M.; ALBAGLI, S. (Org.). Informação e globalização na era do conhecimento. Rio de Janeiro: Editora Campus, 1999. p. 27-57.

MORGAN, K. The Exaggerated Death of Geography: Learning, Proximity and Territorial Innovation Systems. Journal of Economic Geography, v. 4, n. 1, p. 3-21, 2004.

Disponível em: < http://www.donorth.co/appurtenancy/pdfs/morgan_not_death geography.pdf $>$. Acesso em: 14/04/2017.

NEWMAN, M. E. J. Scientific collaboration networks I. Network construction and fundamentals results. Physical Review E, Woodbury, v. 64, 2001. Disponível em: < https://doi:10.1103/PhysRevE.64.016131>. Acesso em: 19/06/2013.

NOHRIA, N.; ECCLES, R. Networks and organizations: structure, form and action. Boston: Harvard Business School Press, 1992.

OLIVEIRA, A. B. A importância do espaço geográfico na construção e funcionamento de redes científicas na área de farmácia em Pernambuco fomentadas pela Política Nacional de Ciência, Tecnologia e Inovação em Saúde (PNCTIS). 2014. 346 f. Tese (Doutorado em Geografia) - Universidade Federal de Pernambuco, Recife, 2014.

OLIVEIRA, A. B.; FERNANDES, A. C. A. O espaço geográfico enquanto temporalidades científicas e técnicas: considerações sobre o impacto da política nacional de ciência, tecnologia e inovação em saúde e o fomento à pesquisa em rede em Pernambuco 2005-2010. Revista Expressões Geográficas, Florianópolis, v. 0, n. 10, p. 1-23, 2015. Disponível em: <http://www.geograficas.cfh.ufsc.br/ arquivo/ed10/ed10 art01.pdf>. Acesso em: 10/04/2018.

PAVITT, K. Sectoral Patterns of Technical Change: Towards a Taxonomy and a Theory. Research Policy, Amsterdam, North-Holland, v. 13, n. 6, p. 343-373, Dec. 1984.

POLANYI, M. The Tacit Dimension. In: PRUSAK, L. Knowledge in Organizations. Nova Iorque: Harper \& Row, 1987.

. The Tacit Dimension. New York: Doubleday, 1966.

- Personal Knowledge: Towards a Post-Critical Philosophy. London: Routledge and Keegan Paul, 1958.

SANTOS, M. A natureza do espaço: técnica e tempo, razão e emoção. 4. ed. São Paulo: EDUSP, 2006. 384 p.

STORPER, M.; VENABLES, A. O burburinho: a força econômica da cidade. In: DINIZ, C.; LEMOS, M. (Org.). Economia e território. Belo Horizonte: UFMG, 2001. p. 21-56. 
TORRE, A.; RALLET, A. Proximity and localization. Regional Studies, Brighton, v. 39, p. 47-59, 2005. https://doi.org/10.1080/0034340052000320842

Allison Bezerra Oliveira - Possui Graduação em Geografia e Especialização em Planejamento Ambiental pela Universidade Estadual do Maranhão, Mestrado e Doutorado em Geografia pela Universidade Federal de Pernambuco. É docente efetivo da Universidade Estadual da Região Tocantina do Maranhão. iD http://orcid.org/0000-0003-0320-5661

Recebido para publicação em 23 de novembro de 2018 Aceito para publicação em 11 de janeiro de 2019 Publicado em 08 de março de 2019 